\title{
CORRECTION TO "PERTURBATIONS OF REGULARIZING MAXIMAL MONOTONE OPERATORS" AND A NOTE ON INJECTIVENESS
}

\author{
BY \\ ERIC SCHECHTER
}

ABSTRACI

Let $A$ be a maximal monotone operator. Let $u_{f}$ be the solution of $f(t) \in u^{\prime}(t)+$ $A u$. We investigate the injectiveness of the mapping $f \mapsto u_{f}$.

In this note we consider the quasiautonomous initial value problem

$$
f(t) \in u^{\prime}(t)+A u(t) \quad(a \leqq t<\infty)
$$

$$
x=u(a)
$$

where $A$ is a maximal monotone operator in a Hilbert space $(H, \mid)$ ). Here $x \in \overline{D(A)}, a \in \mathbf{R}$, and $f: \mathbf{R} \rightarrow H$ is assumed to be locally integrable. For any such $A, x, a, f$, it is well known that (1) has a unique solution $u_{f}:[a, \infty) \rightarrow \overline{D(A)}$. The solution depends continuously on the data, in the following sense:

$$
\left|u_{f}(t)-u_{g}(t)\right| \leqq\left|u_{f}(a)-u_{g}(a)\right|+\int_{a}^{t}|f(s)-g(s)| d s \quad(a \leqq t<\infty)
$$

(see lemma 3.1 in [1]). For later reference, we also note that

$$
\left|u_{f}(t)-u_{g}(t)\right|^{2} \leqq\left|u_{f}(a)-u_{g}(a)\right|^{2}+2 \int_{a}^{t}\left(f(s)-g(s), u_{f}(s)-u_{g}(s)\right) d s
$$

(see inequality (28) on page 65 of [1]). We assume the reader is familiar with the most basic properties of this class of operators and initial value problems. For a brief introduction to this subject see $\$ 6-10$ of [4], or $\$$ II.1-II.4 and III.1-III.2 of [1].

In this paper we shall hold $A$ fixed, and vary $x, a, f, t$. To display dependence on these data, we shall often denote the solution $u(t)$ of $(1)$ by $u_{f}(t, a, x)$. Let $\mathcal{M}$ 
be the set of mappings from $\{(t, a, x) \in \mathbf{R} \times \mathbf{R} \times \overline{D(A)}: t \geqq a\}$, into $\overline{D(A)}$. For each $f \in L_{\mathrm{loc}}^{\mathrm{i}}(\mathbf{R} ; H)$, the function $u_{f}$ is an element of $\mathcal{M}$.

Inequality (2) shows that the mapping $f \mapsto u_{f}$ is continuous from $L_{\mathrm{loc}}^{1}(\mathbf{R} ; H)$ with its usual topology, into $\mathcal{M}$ with the topology of uniform convergence on compact sets. Results in $[5,6]$ show that for certain classes of $A$ 's and $f$ 's, the mapping is still continuous if $L_{\mathrm{loc}}^{\mathrm{loc}}(\mathbf{R} ; H)$ is given a weaker topology. For some classes of $A$ 's and $f$ 's, arguments in [6] and in example 2 of [5] show that the mapping $f \mapsto u_{f}$ is actually a homeomorphism.

Example 3 of [5] asserts that if $H=\mathbf{R}$ and

$$
A(x)= \begin{cases}\operatorname{sign}(x) & \text { when } x \neq 0, \\ {[-1,1]} & \text { when } x=0\end{cases}
$$

then the mapping $f \mapsto u_{f}$ is not injective. That assertion is erroneous, as we shall see later in this note. The brief argument in example 3 of [5] actually shows only that the mapping $f \mapsto u_{f}(\cdot, 0,0)$ is not injective. Clearly, this is a weaker conclusion. But there do indeed exist maximal monotone operators $A$ for which the mapping $f \mapsto u_{f}$ is not injective. This fact will also follow from our main result, below.

Theorem. Let $A$ be a maximal monotone operator in a real Hilbert space $(H,||)$. Let $x_{0} \in D(A)$. Let $H_{0}$ be the closed linear span of the set $D(A)-x_{0}$. Let $P$ be the orthogonal projection of $H$ onto the closed linear subspace $H_{0}$. Let $f_{1}, f_{2} \in L_{\mathrm{loc}}^{1}(\mathbf{R} ; H)$. Then $u_{f_{1}}=u_{f_{2}}$ if and only if $P f_{1}(t)=P f_{2}(t)$ for almost every $t \in \mathbf{R}$.

REMARK. It is easy to show that $H_{0}$ depends only on the set $D(A)$, and not on the particular choice of the element $x_{0}$ in $D(A)$.

PROOF OF THE "IF" PART. Assume $P f_{1}(s)=P f_{2}(s)$ for almost all $s \in \mathbf{R}$. Since $u_{f_{1}}$ and $u_{f_{2}}$ take values in $\overline{D(A)}$, it follows easily that $u_{f_{1}}-u_{f_{2}}$ takes values in $H_{0}$. Hence $u_{f_{1}}(s)-u_{f_{2}}(s)$ is orthogonal to $f_{1}(s)-f_{2}(s)$ for almost all $s$. Now apply (3); it follows that $u_{f_{1}}=u_{f_{2}}$.

PROOF OF THE "ONLY IF" PART. Assume that

$$
P f_{1}(a) \neq P f_{2}(a),
$$

for all $a$ in some set of positive measure. By the vector version of Lebesgue's Theorem [3, theorem III.12.8], almost every point $a \in \mathbf{R}$ is a Lebesgue point of $f_{1}$ and $f_{2}$; that is, a number such that 
(6) $\quad f_{i}(a)$ is defined and $\lim _{h \rightarrow 0} \frac{1}{h} \int_{a}^{a+h}\left|f_{i}(a)-f_{j}(s)\right| d s=0 \quad(j=1,2)$.

Fix some $a \in \mathbf{R}$ satisfying both (5) and (6); it suffices to show that $u_{f_{1}}(\cdot, a, \cdot) \neq u_{f_{2}}(\cdot, a, \cdot)$.

Let $\xi=f_{1}(a)-f_{2}(a)$. It follows from (5) that $\left(\xi, x_{1}-x_{0}\right)$ is nonzero for some $x_{1} \in D(A)$. Define the operator $A+\xi$ by taking $(A+\xi)(x)=A(x)+\xi$ for all $x \in D(A)$. We claim that the operators $A$ and $A+\xi$ are not identical.

Indeed, suppose that $A=A+\xi$. Then $A=A+k \xi$ for all integers $k$. Since $x_{j} \in D(A)$, the set $A x_{j}$ is nonempty; let $y_{j} \in A x_{j}$ for $j=0,1$. Then $y_{j} \in A x_{j}+\theta_{j} k \xi$ if $\theta_{j} \in\{0,1\}$. That is, $\left[x_{i}, y_{j}-\theta_{j} k \xi\right] \in A$. Since $A$ is monotone,

$$
\begin{aligned}
0 & \leqq\left(\left(y_{1}-\theta_{1} k \xi\right)-\left(y_{0}-\theta_{0} k \xi\right), x_{1}-x_{0}\right) \\
& =\left(y_{1}-y_{0}, x_{1}-x_{0}\right)-k\left(\theta_{1}-\theta_{0}\right)\left(\xi, x_{1}-x_{0}\right) .
\end{aligned}
$$

Holding $x_{0}, x_{1}, y_{0}, y_{1}$ fixed, choose $\theta_{0}$ and $\theta_{1}$ so that $\theta_{1}-\theta_{0}=\operatorname{sign}\left(\xi, x_{1}-x_{0}\right)$. Then take $k$ very large; we obtain a contradiction. Thus $A$ and $A+\xi$ are indeed distinct. That is, $A+f_{1}(a)$ and $A+f_{2}(a)$ are distinct.

For any constant $z \in H$, the operator $A+z$ is also maximal monotone, and so it generates a nonexpansive, strongly continuous semigroup $S_{z}$ on $\overline{D(A+z)}=$ $\overline{D(A)}$. The maximal monotone operators are in one-to-one correspondence with their semigroups ([1], theorems 3.1 and 4.1). Since $A+f_{1}(a) \neq A+f_{2}(a)$, it follows that $S_{f_{1}(a)} \neq S_{f_{2}(a)}$.

For $r>0$, let

$$
\delta(r) \equiv \max _{j=1,2} \sup _{0<h \leq r} \frac{1}{h} \int_{a}^{a+h}\left|f_{j}(a)-f_{j}(s)\right| d s .
$$

Then $\delta(r) \downarrow 0$ as $r \downarrow 0$, by our choice of $a$ satisfying (6). For any $x \in \overline{D(A)}$, note that $S_{f(a)}(h) x=u_{\left.f_{f} a\right)}(a+h, a, x)$; hence from (2) we have

$$
\left.\left|S_{f(a)}(h) x-u_{f}(a+h, a, x)\right| \leqq \int_{a}^{a+h}\left|f_{i}(a)-f_{i}(s)\right| d s \leqq h \delta(h)\right) .
$$

This inequality holds for $j=1,2$. Taking the difference of these two results, we obtain

$$
\left|S_{f_{1}(a)}(h) x-S_{f_{2}(a)}(h) x\right| \leqq 2 h \delta(h) \quad \text { for all } x \in \overline{D(A)} \text { and } h \geqq 0 .
$$

Now, let any $y \in \overline{D(A)}$ and $t \in(0, \infty)$ be given. Temporarily fix any positive integer $n$. For $j=0,1,2, \cdots, n$, let $y_{j}=S_{f_{2}(a)}(j t / n) y$. Then 


$$
\begin{aligned}
& \left|S_{f_{1}(a)}(t) y-S_{f_{2}(a)}(t) y\right| \\
& \quad=\left|\sum_{j=1}^{n}\left[S_{f_{1}(a)}(t / n)^{i} S_{f_{2}(a)}(t / n)^{n-j} y-S_{f_{1}(a)}(t / n)^{j-1} S_{f_{2}(a)}(t / n)^{n-j+1} y\right]\right| \\
& \quad \leqq \sum_{j=1}^{n}\left|S_{j_{1}(a)}(t / n) y_{n-j}-S_{f_{2}(a)}(t / n) y_{n-j}\right| \\
& \quad \leqq \sum_{j=1}^{n} 2 \frac{t}{n} \delta\left(\frac{t}{n}\right)=2 t \delta\left(\frac{t}{n}\right)
\end{aligned}
$$

which tends to 0 as $n \rightarrow \infty$. Thus $S_{f_{1}(a)}(t) y=S_{f_{2}(a)}(t) y$ for all $t>0$ and $y \in \overline{D(A)}$, a contradiction. This completes the proof of the theorem.

COROLlary. Let A be a maximal monotone operator in a Hilbert space H. Let $x_{0} \in D(A)$. Then the mapping $f \mapsto u_{f}$, from $L_{\mathrm{loc}}^{1}(\mathbf{R} ; H)$ into $\mathcal{M}$, is injective if and only if the closed linear span of the set $D(A)-x_{0}$ is all of $H$.

REMARKS ON SOME CONSEQUENCES

(1) If $A$ is defined as in (4), then $D(A)=\mathbf{R}$. Hence, by the corollary above, the mapping $f \mapsto u_{f}$ is injective from $L_{\text {loc }}^{1}(\mathbf{R})$ into $\mathcal{M}$.

(2) We easily construct examples in which the mapping $f \mapsto u_{f}$ is not injective. For instance, suppose $A$ is a maximal monotone operator in a Hilbert space $H$, and $\tilde{H}$ is another Hilbert space. Let $B(x)=A(x) \oplus \tilde{H}$ for all $x \in D(A)$. Then $B$ is easily seen to be maximal monotone in $H \oplus \tilde{H}$, with $D(B)=D(A) \subseteq H \subseteq$ $H \oplus \tilde{H}$. If $\tilde{H}$ is not the trivial space $\{0\}$, then $H$ is a proper subspace of $H \oplus \tilde{H}$; and so by the corollary above, the mapping $f \mapsto u_{f}$ for $B$ is not injective.

SOME QUESTIONS FOR FURTHER STUDY

(1) Suppose $H=H_{0}$, so that $f \mapsto u_{f}$ is injective. What topologies on $\mathcal{M}$ and $L_{\text {loc }}^{1}(\mathbf{R} ; H)$ make the inverse map $u_{f} \mapsto f$ continuous? What topologies make it a homeomorphism?

(2) Suppose $H \neq H_{0}$. Then the map $P f \mapsto u_{P f}$ is injective. What topologies make its inverse continuous?

(3) The proof above used the fact that strongly continuous, nonexpansive semigroups on a closed convex subset $C$ of a Hilbert space are in one-to-one correspondence with the maximal monotone operators $A$ satisfying $\overline{D(A)}=C$; That fact seems to be stronger than is really needed for the proof. Indeed, the proof given above does not involve all maximal monotone operators $A$ satisfying $\overline{D(A)}=C$; it only involves those which differ by a constant. Can a more direct proof be given, using less powerful tools and yielding more insight into the map $f \mapsto u_{f}$ ? 
(4) To what extent does the theorem above extend to m-accretive operators in an arbitrary Banach space? It is not clear what should replace the notion of orthogonal projections in that setting. Also, m-accretive operators are not in one-to-one correspondence with their semigroups (pages 295-297 in [2]).

\title{
REFERENCES
}

1. H. Brézis, Opérateurs maximaux Monotones, North-Holland Mathematics Studies 5, Notas de Matemática 50, North-Holland/American Elsevier, 1973.

2. M. G. Crandall and T. M. Liggett, Generation of semi-groups of nonlinear transformations on general Banach spaces, Am. J. Math. 93 (1971), 265-298.

3. N. Dunford and J. T. Schwartz, Linear Operators, I, Wiley, New York, 1957.

4. A. Haraux, Nonlinear Evolution Equations: Global Behavior of Solutions, Springer Lecture Notes in Mathematics 841 (1981).

5. E. Schechter, Perturbations of regularizing maximal monotone operators, Isr. J. Math. $\mathbf{4 3}$ (1982), 49-61.

6. E. Schechter, Evolution generated by semilinear dissipative plus compact operators, Trans. Am. Math. Soc. 275 (1983), 297-308.

\author{
Mathematics Department \\ VANDERBILT UNIVERSITY \\ NASHVILLE, TN 37235 USA
}

